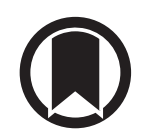

CrossMark

\title{
A regimen containing bedaquiline and delamanid compared to bedaquiline in patients with drug-resistant tuberculosis
}

\author{
Olatunde Olayanju ${ }^{1,3}$, Aliasgar Esmail $^{1,3}$, Jason Limberis ${ }^{1}$ and Keertan Dheda ${ }^{1,2}$ \\ Affiliations: ${ }^{1}$ Centre for Lung Infection and Immunity, Division of Pulmonology, Dept of Medicine, and UCT \\ Lung Institute and South African MRC/UCT Centre for the Study of Antimicrobial Resistance, Division of \\ Pulmonology, University of Cape Town, Cape Town, South Africa. ${ }^{2}$ Faculty of Infectious and Tropical Diseases, \\ Dept of Immunology and Infection, London School of Hygiene and Tropical Medicine, London, UK. ${ }^{3}$ Co-first \\ author.
}

Correspondence: Keertan Dheda, Centre for Lung Infection and Immunity Unit, Division of Pulmonology, Dept of Medicine University of Cape Town, H46.41 Old Main Building, Groote Schuur Hospital, Observatory, 7925 South Africa. E-mail: keertan.dhedaQuuct.ac.za

@ERSpublications

A bedaquiline-delamanid combination regimen in drug-resistant tuberculosis patients with poor prognostic factors showed comparable efficacy and safety to those in a bedaquiline-based regimen http://bit.ly/32j7Fyo

Cite this article as: Olayanju O, Esmail A, Limberis $\mathrm{J}$, et al. A regimen containing bedaquiline and delamanid compared to bedaquiline in patients with drug-resistant tuberculosis. Eur Respir J 2020; 55: 1901181 [https://doi.org/10.1183/13993003.01181-2019].

\section{ABSTRACT}

There are limited data on combining delamanid and bedaquiline in drug-resistant tuberculosis (DR-TB) regimens. Prospective long-term outcome data, including in HIV-infected persons, are unavailable.

We prospectively followed up 122 South African patients (52.5\% HIV-infected) with DR-TB and poor prognostic features between 2014 and 2018. We examined outcomes and safety in those who received a bedaquiline-based regimen $(n=82)$ compared to those who received a bedaquiline-delamanid combination regimen $(n=40)$.

There was no significant difference in 6-month culture conversion $(92.5 \%$ versus $81.8 \%$; $=0.26)$ and 18 -month favourable outcome rate $(63.4 \%$ versus $67.5 \% ; \mathrm{p}=0.66)$ in the bedaquiline versus the bedaquiline-delamanid combination group, despite the latter having more advanced drug resistance $(3.7 \%$ versus $22.5 \%$ resistant to at least five drugs; $\mathrm{p}=0.001)$ and higher pre-treatment failure rates $(12.2 \%$ versus $52.5 \%$ with pre-treatment multidrug-resistant TB therapy failure; $\mathrm{p}<0.001)$. Although the proportion of prolongation of the QT interval corrected using Fridericia's formula was higher in the combination group ( $>60 \mathrm{~ms}$ from baseline $(\mathrm{p}=0.001)$ or $>450 \mathrm{~ms}$ during treatment $(\mathrm{p}=0.001)$ ), there were no symptomatic cases or drug withdrawals in either group. Results were similar in HIV-infected patients.

A bedaquiline-delamanid combination regimen showed comparable long-term safety compared to a bedaquiline-based regimen in patients with DR-TB, irrespective of HIV status. These data inform regimen selection in patients with DR-TB from TB-endemic settings. 


\section{Introduction}

Drug-resistant tuberculosis (DR-TB) constitutes a threat to TB control globally. In 2017, despite high rates of underreporting and underdiagnosis, there was a 5\% increase in the absolute number of DR-TB cases detected compared to 2016 [1, 2]. Treatment outcomes of multidrug-resistant (MDR)-TB have also remained poor, with a treatment success rate of 55\% reported in 2017 [3, 4]. However, this is expected to improve given that newer and repurposed drugs have now been recommended by the World Health Organization (WHO) for the treatment of MDR-TB [5]. Indeed, studies and regimens containing bedaquiline and linezolid have shown impressive efficacy in clinical trials and real-life programmatic use [6-10].

Nevertheless, even with the use of these newer drugs, successful outcomes and treatment options are limited in patients with fluoroquinolone-resistant and extensively drug-resistant TB (XDR)-TB [7]. Thus, frequently there is a difficulty in constituting an effective treatment regimen [2, 11-14]. Patients who have had previous exposure to second-line TB medication, developed resistance to multiple drugs, developed intolerance due to adverse events [15] or failed therapy on bedaquiline-based regimens are all predisposed to having a poor prognosis. In this group of patients, it is challenging to constitute a treatment regimen containing at least four to five likely effective drugs (drugs to which the isolate was susceptible and/or to which patients had $<1$ month or no previous exposure) without simultaneously including bedaquiline and delamanid. The latter, a group $\mathrm{C}$ drug, remains widely used in clinical practice, although there is currently limited evidence about the efficacy of delamanid for the treatment of MDR-TB [16-18].

Thus, in appropriate patients with high-level resistance and/or poor prognosis, there is an increasing need to use a bedaquiline-delamanid combination. However, the potential for synergistic corrected QT interval (QTc) prolongation from both drugs, predisposing patients to cardiac arrhythmias and sudden death, has created unease over their inclusion in the same treatment regimen [4, 19-21]. Despite this concern and more widespread use, few studies have reported on the concurrent use of delamanid and bedaquiline and have mostly described early safety and efficacy of the combination in retrospective cohorts [22, 23]. There are, however, no prospective and long-term data addressing safety, and none addressing the efficacy of the combination regimen in patients with poor prognosis from a TB-endemic setting. Furthermore, there are limited data about use of the bedaquiline-delamanid combination in HIV co-infected patients and those with high-level resistance, e.g. patients with XDR-TB. Another major limitation has been the lack of comparative data from a bedaquiline only control group, so that the net effect of delamanid safety (and QT prolongation) over that of bedaquiline could be ascertained. We therefore compared the treatment outcomes and adverse event profiles of DR-TB patients with poor prognostic features on a bedaquiline-based regimen to those who had received the bedaquiline-delamanid combination.

\section{Methods}

Study design and participants

We prospectively recruited, through an ongoing clinical registry, microbiologically confirmed MDR-TB patients who were admitted to Brooklyn Chest Hospital (Cape Town, South Africa), the designated treatment centre for DR-TB in the Western Cape province in South Africa. However, a per protocol analysis plan was only formulated retrospectively. All patients were admitted between January 2014 and April 2018. Patients were included in the study if their drug susceptibility and testing performed prior to recruitment showed resistance to at least rifampicin and isoniazid. Sputum drug susceptibility and testing was repeated on a monthly basis following treatment initiation, to monitor treatment progress. Each patient received either a bedaquiline-based or a bedaquiline-delamanid combination regimen. Medications were administered by trained healthcare workers to patients during admission and on an outpatient basis after discharge from the hospital.

Adverse events were reported by a medically qualified healthcare worker, using a pharmacovigilance report form provided for each patient. Each patient underwent ECG before treatment initiation and on a minimum of a monthly basis after treatment began. QT interval was corrected using Fridericia's formula (QTcF), and values $>450 \mathrm{~ms}$ qualified a patient for closer review by the attending physician. Patients' demographic and clinical data were captured by a trained researcher, and laboratory reports were regularly updated on a dedicated database using a standard case report form. Written consent was obtained from every participant, and ethical clearance was obtained from the University of Cape Town Human Research Ethics Committee.

\section{Treatment groups}

All patients were assigned a treatment group based on the drugs that constituted the backbone of their regimen. Bedaquiline was the backbone in the bedaquiline-based regimen group, while the backbone of the regimen in the second group was a combination of bedaquiline and delamanid (bedaquiline-delamanid), administered concurrently. Indications for patients to be given the combination therapy were the inability 
to construct an effective regimen (at least four likely effective drugs) for the patient due to extensive drug resistance patterns or adverse events, the strengthening of a regimen due to late conversion, extensive lung disease and previous failure on a bedaquiline-based regimen. Medications were individualised for patients based on their phenotypic drug susceptibility test results.

\section{Outcomes}

Culture conversion was defined as two consecutive negative sputum culture results, taken at least 30 days apart (one missing or contaminated culture was allowed between negative cultures, and inability to produce sputum was considered to be a negative result). Culture conversion status was compared between the two groups at 2 months, 6 months and 12 months following treatment initiation. In the first 6 months of therapy, changes in the QTcF interval from baseline values were also compared between the two groups to establish how it is impacted by the treatment regimens. At follow-up censor date, which was a minimum of 18 months after initiation of treatment, patients were assigned to have had a favourable outcome if they had completed treatment or were cured, whereas those who died during treatment, failed to achieve culture conversion or were lost to follow-up were said to have had an unfavourable outcome.

\section{Statistical analysis}

The impact of delamanid was determined by comparative analysis of demographics, clinical characteristics and treatment outcomes. Qualitative and quantitative variables were reported in terms of percentages and median interquartile range (IQR). Quantitative and qualitative variables were compared using the MannWhitney U-test and Chi-squared test or Fisher's exact test, respectively. A univariate Cox proportional hazard model was used to estimate the relationship between independent variables (demographic and clinical characteristics) and the development of unfavourable outcomes and having at least one QTc value of $>450 \mathrm{~ms}$; variables with a $\mathrm{p}$-value $<0.3$ were included in the multivariate model. A p-value $<0.05$ was taken as statistically significant. Kaplan-Meier curves for the probability of achieving an unfavourable outcome and the proportion of patients with culture positivity were estimated considering the duration between the day of treatment initiation and the follow-up censor date. Comparisons between strata (bedaquiline-based therapy and bedaquiline-delamanid combination therapy) were undertaken using a log-rank test. Statistical analysis was performed using IBM SPSS Statistics (Version 25.0; IBM Corp., Armonk, NY, USA).

\section{Results}

\section{Demographic and clinical characteristics}

In this study, a total of 122 culture-confirmed MDR-TB patients were enrolled into either a bedaquiline-based regimen group or a bedaquiline-delamanid combination regimen group. Median (IQR) age at admission was 34 (27-42) years; they were on admission for a median (IQR) 161 (102-230) days and $74(60.7 \%)$ were male. The median (IQR) weight at admission was $51.8(43.8-59.0) \mathrm{kg}, 64(52.5 \%)$ patients were HIV infected with a median (IQR) CD4 count of $154(57-332)$ cells $\mu \mathrm{L}^{-1}$ and they were all on antiretroviral therapy. Isolates from 11 (9\%) patients were fully MDR-TB, 25 (20.5\%) patients had further resistance to either a fluoroquinolone or a second-line injectable (pre-XDR-TB), while 86 (70.5\%) patients were resistant to both (XDR-TB).

The $82(67.2 \%)$ patients in the group that received the bedaquiline-based regimen were hospitalised for a median (IQR) of 155 (93-210) days. Patient demographics and clinical characteristics are outlined in table 1. They received a median (IQR) of 8 (7-9) medications in the regimen, which comprised bedaquiline, clofazimine, levofloxacin and linezolid as the major components. Other drugs and the proportion of patients who received them are outlined in table 2 . All patients received clofazimine or a fluoroquinolone, which are known QTc-prolonging drugs. 23 (28.1\%) patients received both drugs, 55 (67.1\%) patients received only clofazimine, and three (3.7\%) patients received only moxifloxacin in their regimen.

In the bedaquiline-delamanid combination regimen group, 40 (32.8\%) patients were enrolled and they were hospitalised for a median (IQR) of 204 (124-295) days. 29 (72.5\%) patients had been previously treated for $\mathrm{TB}$, and nine (22.5\%) were resistant to at least five drugs; other markers of disease severity are highlighted in table 1 . They received a median (IQR) of $10(8-11)$ medications in the regimen, which was significantly more than the medications received in the bedaquiline-based regimen. The major medications in this regimen were delamanid, bedaquiline, clofazimine, levofloxacin and linezolid. 37 (92.5\%) patients in this group received at least one of either clofazimine or moxifloxacin. 14 (35.0\%) patients received both drugs, 21 (52.5\%) patients received only clofazimine, while two (5.0\%) patients received only moxifloxacin in their regimen.

\section{Markers of disease severity}

There were significantly more patients $(72.5 \%)$ in the bedaquiline-delamanid combination regimen group with previous exposure to TB treatment compared to those in the bedaquiline-based regimen $(48.8 \% ; \mathrm{p}=0.01)$. 
TABLE 1 Comparison of demographic and clinical characteristics between patients in the bedaquiline and the combination regimen groups

Patients receiving bedaquiline-based regimen
Patients receiving bedaquiline-delamanid p-value combination regimen

\begin{tabular}{|c|c|c|c|}
\hline Subjects $n$ & 82 & 40 & \\
\hline Age years & $33(28-42)$ & $34(27-42)$ & 0.04 \\
\hline Males & $50(61.0)$ & $24(60.0)$ & 0.92 \\
\hline Weight at admission $\mathbf{k g}$ & $51.8(45.6-58.3)$ & $51.8(43.3-60.8)$ & 0.36 \\
\hline Number of medications & $8(7-9)$ & $10(8-11)$ & 0.001 \\
\hline Five likely effective medications & $59(72)$ & $26(65)$ & 0.43 \\
\hline Number of adverse events & $2(1-4)$ & $3(2-4)$ & 0.51 \\
\hline XDR-TB & $67(81.7)$ & 19 (47.5) & $<0.001$ \\
\hline Pre-XDR-TB & 10 (12.2) & 15 (37.5) & $<0.001$ \\
\hline MDR-TB & $5(6.1)$ & $6(15)$ & $<0.001$ \\
\hline \multicolumn{4}{|l|}{ Markers of disease severity } \\
\hline Patients with weight $<50 \mathrm{~kg}$ & $34(41.5)$ & 19 (47.5) & 0.53 \\
\hline $\begin{array}{l}\text { Patients with previous MDR-TB treatment } \\
\text { failure }\end{array}$ & 10 (12.2) & $21(52.5)$ & $<0.001$ \\
\hline Patients with $\mathrm{CD} 4<200$ cells $\mu \mathrm{L}^{-1}$ & $26(31.7)$ & $10(29.4)$ & 0.19 \\
\hline Diabetic patient & $2(2.4)$ & $1(2.5)$ & 0.98 \\
\hline Patients resistant to $\geqslant 5$ drugs & $3(3.7)$ & $9(22.5)$ & 0.001 \\
\hline $\begin{array}{l}\text { Patients either resistant to } \geqslant 5 \text { drugs or with } \\
\text { previous treatment failure }\end{array}$ & $13(15.9)$ & $23(57.5)$ & $<0.001$ \\
\hline Smear grade $>2$ pluses & $14(17.1)$ & $8(20.0)$ & 0.69 \\
\hline Time to culture positivity $\leqslant 7$ days & $9(10.9)$ & $7(17.5)$ & 0.32 \\
\hline \multicolumn{4}{|l|}{ Treatment outcome } \\
\hline Favourable outcome & $52(63.4)$ & $27(67.5)$ & 0.66 \\
\hline Unfavourable outcome & $30(36.6)$ & 13 (32.5) & 0.66 \\
\hline
\end{tabular}

Data are presented as median (interquartile range) or $\mathrm{n}(\%)$, unless otherwise stated. XDR-TB: extensively drug-resistant tuberculosis; MDR-TB: multidrug-resistant tuberculosis.

More patients in the combination therapy group had previously failed TB treatment compared to those in the bedaquiline group (52.5\% versus $12.2 \%$; $<0001)$. The bedaquiline-delamanid combination regimen group also had significantly more patients with resistance to at least five drugs (22.5\% versus $3.7 \%$; $\mathrm{p}=0001)$. Other markers of disease severity, including HIV infection, microbial burden and weight $<50 \mathrm{~kg}$ at admission were mostly higher in the bedaquiline-delamanid combination regimen group (table 1).

\section{Culture conversion}

In the bedaquiline-based regimen group, 52 (63.4\%) patients were culture-positive at recruitment, and $92.5 \%$ of those with laboratory results had achieved culture conversion by 6 months of treatment. Of the 42 HIV-infected patients in this group, 23 (54.8\%) were culture-positive at recruitment, and 93.8\% of those with laboratory results had achieved culture conversion by 6 months of treatment.

In the bedaquiline-delamanid combination regimen, 26 (65\%) patients were culture-positive at recruitment, and $81.8 \%$ of those with laboratory results had achieved culture conversion by 6 months of treatment. Of the $22 \mathrm{HIV}$-infected patients in this group, 13 (59.1\%) were culture-positive at recruitment, and $83.3 \%$ of those with laboratory results had achieved culture conversion by 6 months of treatment. Comparisons of culture conversion rates in both groups are outlined in supplementary table S1. In time-to-event analysis, there were more patients in the bedaquiline-delamanid combination group with culture positivity compared to those in the bedaquiline group by the end of month 15 ( $p=0.04$; figure 1 ).

\section{Efficacy of the treatment regimens}

In the bedaquiline-based regimen group, $52(63.4 \%)$ patients achieved a favourable outcome while the remaining patients had an unfavourable outcome by the end of the follow-up period. 29 (69.1\%) out of 42 
TABLE 2 Drugs used in the bedaquiline-based and bedaquiline-delamanid combination regimen and the proportion of patients who received them

Patients receiving bedaquiline-based regimen
Patients receiving bedaquiline-delamanid combination regimen

\begin{tabular}{lccc}
\hline Subjects n & 82 & 40 & \\
Kanamycin & $16(19.5)$ & $8(20)$ & $33(82.5)$ \\
Terizidone & $75(91.5)$ & $37(92.5)$ & 0.95 \\
Pyrazinamide & $80(97.6)$ & $31(77.5)$ & 0.15 \\
Para-aminosalicylic acid & $75(91.5)$ & $5(12.5)$ & 0.19 \\
Meropenem & $0(0)$ & $37(92.5)$ & $\mathrm{N} / \mathrm{A}$ \\
Any fluoroquinilone & $81(98.8)$ & $16(40)$ & 0.07 \\
Moxifloxacin & $26(31.7)$ & $33(82.5)$ & 0.37 \\
Levofloxacin & $81(98.8)$ & $36(90)$ & 0.001 \\
Linezolid & $67(81.7)$ & $20(50)$ & 0.24 \\
High-dose isoniazid & $34(41.5)$ & $13(32.5)$ \\
Ethionamide & $27(32.9)$ & $14(35)$ & 0.37 \\
Ethambutol & $38(46.3)$ & $35(87.5)$ & $3(7.5)$ \\
Clofazimine & $78(95.1)$ & $7(100)$ \\
Capreomycin & $7(8.5)$ & $40(100)$ \\
Bedaquiline & $82(100)$ & 0.96 \\
Delamanid & $0(0)$ & 0.13 \\
\end{tabular}

Data are presented as $\mathrm{n}(\%)$, unless otherwise stated. N/A: not applicable.

HIV-infected patients in this group also achieved a favourable outcome. In the bedaquiline-delamanid combination regimen group, $27(67.5 \%)$ patients achieved a favourable outcome while the remaining patients had an unfavourable outcome by the end of the follow-up period. 15 (68.2\%) out of 22 HIV-infected patients in this group also achieved a favourable outcome. There was no significant difference in the favourable outcome rate between the two groups even when they were stratified by resistance patterns (table 1 and supplementary table S2). In time-to-event analysis, there was no difference in the probability of achieving an unfavourable outcome between the two groups $(\mathrm{p}=0.54)$ (figure 1). Regression analysis showed that moxifloxacin (HR 1.023; $\mathrm{p}=0.89$ ) and clofazimine (HR 0.711; $\mathrm{p}=0.35$ ), which were the other QTcF-prolonging drugs used in both regimens, did not cause a predisposition to an unfavourable outcome (table 3). It also suggested that time to sputum culture positivity of $<7$ days $(\mathrm{HR}=2.712 ; \mathrm{p}=0.006)$ and resistance to at least five drugs $(\mathrm{HR}=2.173 ; \mathrm{p}=0.08)$ are independent predictors of an unfavourable outcome (table 3 ).

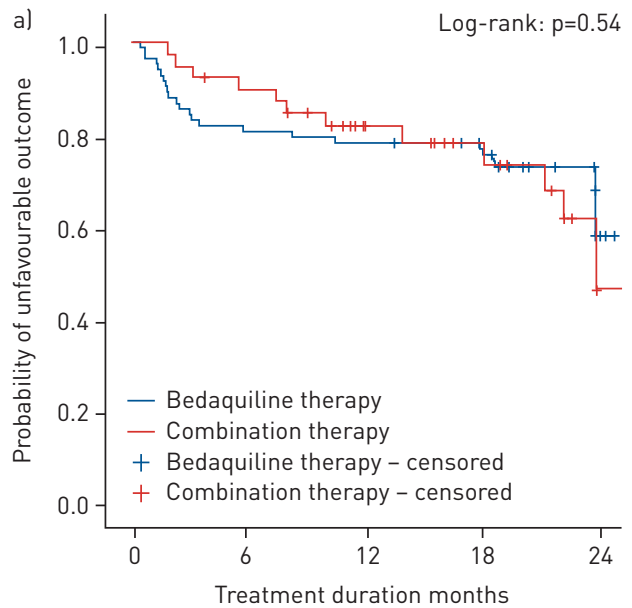

At risk $n$

$\begin{array}{llllll}\text { B } & 82 & 14 & 12 & 11 & 4\end{array}$

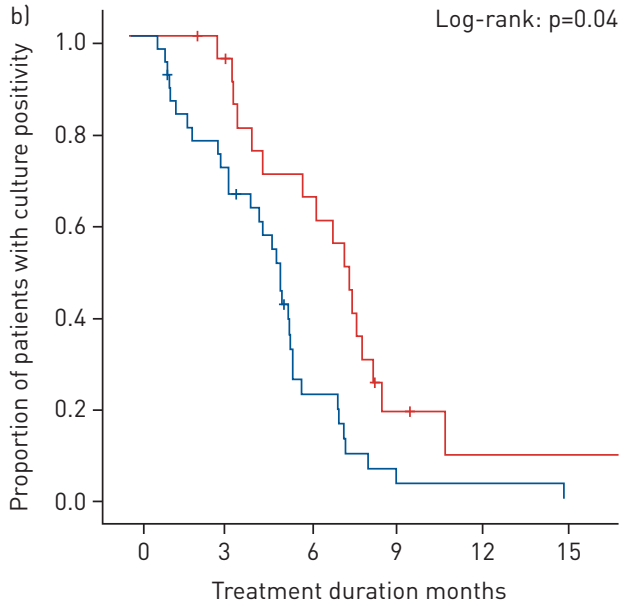

At risk $n$

\begin{tabular}{|c|c|c|}
\hline B & 52 & 46 \\
\hline
\end{tabular}

FIGURE 1 Kaplan-Meier estimates for al the probability of achieving an unfavourable outcome and b) the proportion of patients with culture positivity by month 15 in patients who received bedaquiline-based regimen (B) versus a bedaquiline-delamanid combination regimen (BD). 


\begin{tabular}{|c|c|c|}
\hline Variables & $\begin{array}{c}\text { Hazard ratio } \\
\text { (95\% CI) }\end{array}$ & p-value \\
\hline \multicolumn{3}{|l|}{ Univariate analysis } \\
\hline Age years & $0.997(0.967-1.028)$ & 0.85 \\
\hline Males & $1.231(0.662-2.289)$ & 0.51 \\
\hline Weight kg & $0.986(0.960-1.014)$ & 0.33 \\
\hline Age at admission $<50$ years & $1.137(0.624-2.070)$ & 0.68 \\
\hline HIV infection & $1.181(0.874-1.595)$ & 0.28 \\
\hline CD4 count cells $\mu \mathrm{L}^{-1}$ & $1.000(0.998-1.002)$ & 0.84 \\
\hline Previous TB treatment & $1.013(0.556-1.848)$ & 0.97 \\
\hline Previous treatment failures & $1.367(0.580-3.223)$ & 0.48 \\
\hline Days of admission & $1.000(0.997-1.002)$ & 0.87 \\
\hline Clofazimine treatment & $0.505(0.122-2.090)$ & 0.35 \\
\hline Delamanid treatment & $0.877(0.627-1.225)$ & 0.44 \\
\hline Moxifloxacin treatment & $1.023(0.743-1.408)$ & 0.89 \\
\hline Levofloxacin treatment & $0.968(0.473-1.980)$ & 0.93 \\
\hline Any fluoroquinolone & $0.897(0.123-6.555)$ & 0.92 \\
\hline Linezolid treatment & $0.959(0.426-2.157)$ & 0.92 \\
\hline Bedaquiline-delamanid treatment & $0.814(0.416-1.593)$ & 0.55 \\
\hline Number of medications & $1.112(0.935-1.322)$ & 0.23 \\
\hline Number of adverse events & $1.026(0.898-1.171)$ & 0.71 \\
\hline Five likely effective drugs & $0.840(0.589-1.196)$ & 0.33 \\
\hline Resistant to $\geqslant 5$ drugs & $2.173(0.900-5.246)$ & 0.08 \\
\hline TTP $<7$ days & $2.712(1.331-5.522)$ & 0.006 \\
\hline Smear grade & $1.583(0.779-3.216)$ & 0.20 \\
\hline \multicolumn{3}{|l|}{ Multivariate analysis } \\
\hline HIV infection & $1.940(0.791-2.751)$ & 0.22 \\
\hline Isolate resistant to $\geqslant 5$ drugs & $1.940(0.787-4.779)$ & 0.15 \\
\hline TTP $<7$ days & $2.681(1.196-6.011)$ & 0.02 \\
\hline Number of medications & $1.144(0.957-1.368)$ & 0.14 \\
\hline Smear grade & $1.084(0.489-2.403)$ & 0.84 \\
\hline
\end{tabular}

Variables with $p$-values of $<0.3$ were included in the multivariate model. TB: tuberculosis; TTP: time to culture positivity. ${ }^{\#}: \mathrm{n}=122$.

\section{Adverse events}

In the bedaquiline-based regimen group, $73(89.0 \%)$ patients reported a total of 250 adverse events, each patient reporting a median (IQR) of 2 (1-4) adverse events in the course of treatment. The most commonly reported adverse events were hearing loss (50.0\%), most likely from previous second-line injectable treatment, elevated liver enzymes (28\%) with median (IQR) alanine aminotransferase (ALT) of $112 \mathrm{U} \cdot \mathrm{L}^{-1}(81-173)$ in affected patients, anaemia $(34.1 \%)$, peripheral neuropathy $(22.0 \%)$ and vomiting (24.4\%). ALT elevation in the HIV-infected patients in this group was by a median (IQR) of $107 \mathrm{U} \cdot \mathrm{L}^{-1}$ (71-154).

In the bedaquiline-delamanid combination regimen group, 37 (92.5\%) patients reported a total of 125 adverse events, each patient reporting a median (IQR) of 3 (2-4) adverse events in the course of treatment. The most commonly reported adverse events in this group were hearing loss (45\%), elevated liver enzymes (32.5\%) with median (IQR) ALT of $111 \mathrm{U} \cdot \mathrm{L}^{-1}$ (85-155), anaemia (37.5\%) and peripheral neuropathy (30.0\%). ALT elevation in the HIV-infected patients in this group was by a median (IQR) of $133 \mathrm{U} \cdot \mathrm{L}^{-1}$ (91-155), which was essentially similar to those in the bedaquiline regimen $(p=0.34)$. There were no significant differences in the occurrence of adverse events reported in the two groups except for psychosis, which was likely associated with higher rates of simultaneous use of terizidone and high-dose isoniazid in the bedaquiline-delamanid combination regimen group. Other adverse events and the proportion of patients who had them are reported in table 4.

\section{QTCF interval changes}

The median (IQR) baseline QTcF value for the bedaquiline-based regimen group was $408 \mathrm{~ms}$ (388-425). In the first 6 months of treatment, there was a maximum QTcF prolongation of median (IQR) $27 \mathrm{~ms}$ (13-42) from the baseline values, and only six (7.3\%) patients had a QTcF prolongation $>60 \mathrm{~ms}$ from 
TABLE 4 Adverse events reported by patients who received bedaquiline-based regimen and those who received bedaquiline-delamanid combination regimen

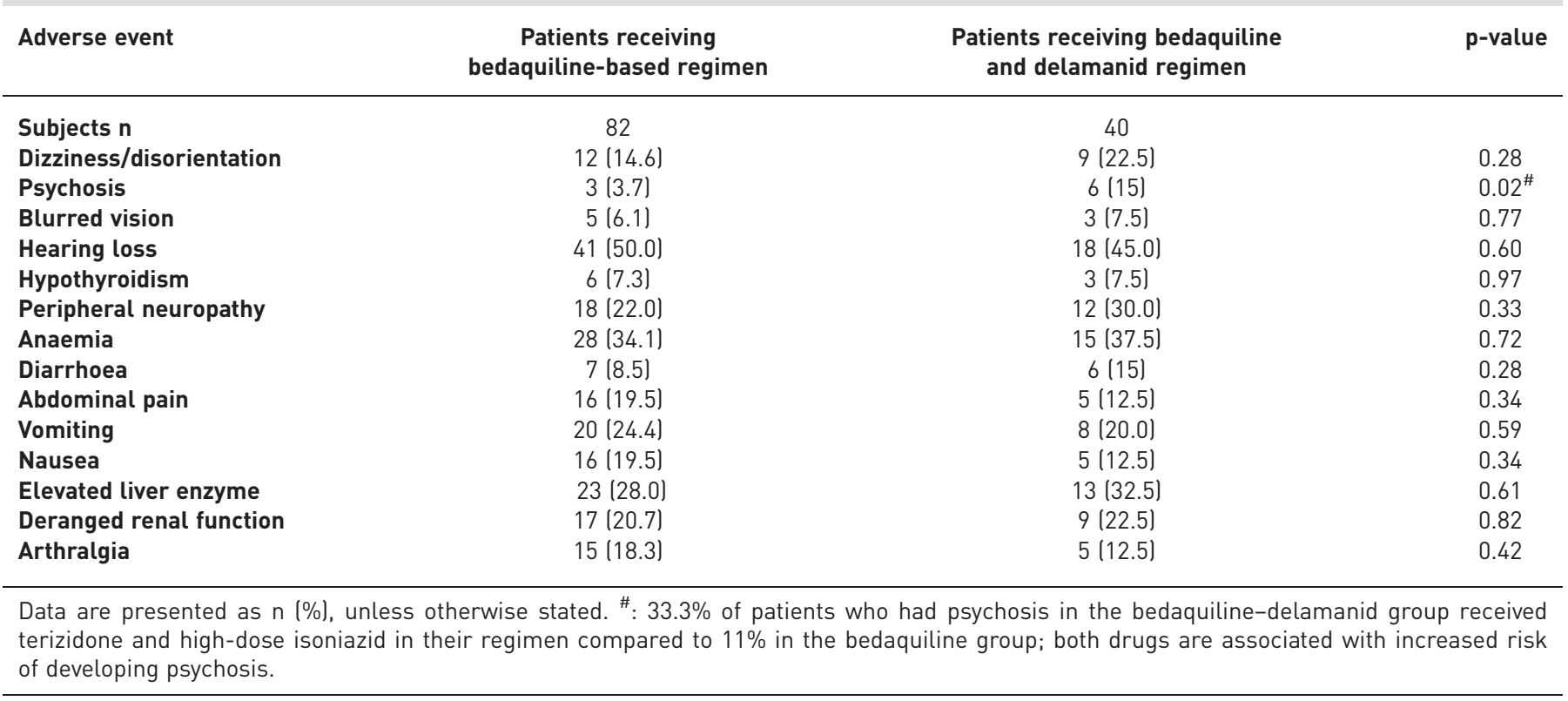

baseline values. 16 (19.5\%) patients in this group also had at least one QTcF value $>450 \mathrm{~ms}$ in the course of treatment, but none of them reached the threshold limit of $500 \mathrm{~ms}$ or had bedaquiline discontinued due to changes in QTcF values (table 5).

The median (IQR) baseline QTcF value for the bedaquiline-delamanid regimen group was 419 (389436) $\mathrm{ms}$. In the first 6 months of treatment, there was a maximum QTcF prolongation of median (IQR) 23

TABLE 5 QTCF profiles of patients who received bedaquiline-based regimen and those who received bedaquiline-delamanid combination regimen in the whole cohort and in HIV-infected patients

Variable

Patients receiving bedaquiline-based regimen

\section{Whole cohort $\mathrm{n}$}

Baseline QTcF

Maximum QTcF change from baseline

Patients with QTcF increment from baseline $>60 \mathrm{~ms}$

Patients with at least one QTcF $>450 \mathrm{~ms}$

Patients with at least one QTcF $>500 \mathrm{~ms}$

HIV-infected patients $\mathbf{n}$

Baseline QTcF

QTcF change from baseline

Patients with QTcF difference $>60 \mathrm{~ms}$

Patients with QTcF $>450 \mathrm{~ms}$

Patients with QTcF $>500 \mathrm{~ms}$

HIV-uninfected patients $\mathbf{n}$

Baseline QTcF

QTcF change from baseline

Patients with QTcF difference $>60 \mathrm{~ms}$

Patients with QTcF > $450 \mathrm{~ms}$

Patients with QTcF $>500 \mathrm{~ms}$

82

$408(388-425)$
$27(13-42)$
$6(7.3)$

16 (19.5)

0 (0)

42

407 (385-428)

$32(14-44)$

5 (11.9)

$11(26.2)$

$0(0)$

40

409 (394-419)

22 (12-37)

1
5
0
Patients receiving bedaquiline-delamanid combination therapy

Data are presented as median (interquartile range) or $\mathrm{n}(\%)$, unless otherwise stated. QTcF: QT interval corrected using Fridericia's formula; N/A: not applicable. 
(8-54) $\mathrm{ms}$ from the baseline values, and only seven (20.6\%) patients had a QTcF prolongation $>60 \mathrm{~ms}$ from baseline values. $15(44.1 \%)$ patients in this group also had at least one QTcF value $>450 \mathrm{~ms}$ in the course of treatment, none of them reached the threshold limit of $500 \mathrm{~ms}$ and neither bedaquiline nor delamanid was discontinued in any of the patients due to changes in QTcF values (table 5).

There was no definitive pattern to the changes (increase or decrease) in QTcF values observed over a period of 6 months (supplementary figure S1), but there were more patients in the bedaquiline-delamanid regimen group who reported a QTcF prolongation $>60 \mathrm{~ms}$ from baseline values $(\mathrm{p}<0.001)$ and at least one QTcF value $>450 \mathrm{~ms}(\mathrm{p}<0.001)$ in the course of treatment. Increasing age $(\mathrm{HR}=1.039 ; \mathrm{p}=0.04)$ and the use of delamanid $(\mathrm{HR}=3.504 ; \mathrm{p}=003)$ were independent predictors of having at least one QTcF value $>450 \mathrm{~ms}$ (table 6). There were, however, no cardiac symptoms necessitating the withdrawal of delamanid from the treatment regimen from any of the affected patients.

\section{Discussion}

This is the first prospective study comparing long-term treatment outcomes and safety data in DR-TB patients who received bedaquiline versus bedaquiline-delamanid combination therapy. The main findings of the study were as follows. 1) Combination therapy was associated with significant QTcF prolongation from baseline values but there were no patients who became symptomatic from a cardiovascular point of view (syncope, collapse, arrhythmia, hypotension, etc.) or reached the threshold limit of $500 \mathrm{~ms}$ that would have necessitated recommended withdrawal of either bedaquiline or delamanid (despite the concomitant use of other QT-prolonging drugs). 2) Bedaquiline-delamanid combination therapy was associated with modestly good culture conversion and favourable outcome rates (comparable to a bedaquiline-based regimen) despite being used in patients from a poorer prognostic category, i.e. a high proportion of patients who had previously been declared therapeutically destitute, and/or whose isolates were resistant to at least five drugs. 3) These findings were broadly similar in HIV-infected patients.

Thus, our data support the use of the combination in patients in whom there is a difficulty in constituting a regimen with at least four effective drugs. Our data are concordant with two recent publications, which

TABLE 6 Cox proportional hazard model for having at least one QTc value $>450 \mathrm{~ms}$

\begin{tabular}{llc} 
Variables & Hazard ratio & p-value \\
& \multicolumn{1}{l}{$(\mathbf{9 5 \%} \mathbf{C l})$} & \\
\hline Univariate analysis & & 0.07 \\
Age years & $1.031(0.998-1.066)$ & 0.61 \\
Males & $1.221(0.570-2.614)$ & 0.24 \\
Weight kg & $0.980(0.948-1.014)$ & 0.12 \\
Age at admission <50 years & $0.427(0.146-1.248)$ & 0.91 \\
HIV infection & $1.043(0.504-2.158)$ & 0.63 \\
Previous TB treatment & $1.195(0.579-2.466)$ & 0.56 \\
Previous treatment failures & $1.304(0.536-3.173)$ & 0.06 \\
Days of admission & $1.002(1.000-1.004)$ & 0.61 \\
Clofazimine treatment & $0.731(0.220-2.426)$ & 0.001 \\
Delamanid treatment & $3.668(1.712-7.859)$ & 0.48 \\
Moxifloxacin treatment & $1.306(0.619-2.757)$ & 0.24 \\
Levofloxacin treatment & $1.564(0.748-3.271)$ & 0.28 \\
Linezolid treatment & $0.605(0.244-1.501)$ & 0.38 \\
Number of medications & $1.104(0.884-1.380)$ & 0.77 \\
Number of adverse events & $0.976(0.832-1.145)$ & 0.16 \\
Five likely effective drugs & $2.025(0.761-5.386)$ & 0.73 \\
TTP <7 days & $0.777(0.184-3.282)$ & 0.38 \\
SMG >2 pluses & $1.504(0.609-3.714)$ & \\
Multivariate analysis & & 0.05 \\
Five likely effective drugs & $3.167(0.995-10.08)$ & 0.04 \\
Age years & $1.039(1.000-1.078)$ & 0.003 \\
Delamanid treatment & $3.504(1.544-7.954)$ & 0.22 \\
Linezolid treatment & $0.539(0.200-1.454)$ & 0.55 \\
Levofloxacin treatment & $1.357(0.494-3.730)$ & 0.46 \\
Days of admission & $1.001(0.998-1.004)$ & 0.23 \\
Weight kg & $0.975(0.937-1.015)$ & \\
& & \\
\hline
\end{tabular}

QTc: corrected QT interval; TB: tuberculosis; TTP: time to culture positivity; SMG: sputum smear grade. 
also confirmed the safety profile of the bedaquiline-delamanid combination $[23,24]$. However, there are several important strengths and incremental contributions of our study findings. In our study we were able to confirm long-term safety and safety in HIV-infected persons (which has always remained the concern given their higher frequency of adverse drug events in general), and importantly we were able to discern the incremental QT prolongation effect of delamanid within the combination when compared to a bedaquiline-only group. This should allay anxiety amongst clinicians who often need to include delamanid in treatment regimens but are concerned by the WHO guidelines, which express reservations about the safety of simultaneous use of these drugs [4].

Other QT-prolonging drugs like clofazimine and moxifloxacin were used substantially, and to a similar extent, in both groups and thus we could account for their effect when comparisons were made. Nevertheless, ECG monitoring is still required when the combination is used, especially together with other QT-prolonging drugs, given that discontinuation of drugs has been documented in a few patients [4]. However, our findings support the notion that this is not a major issue and is uncommon.

We were also able to evaluate the long-term efficacy, in terms of outcomes, in the bedaquiline-only and combination groups. It is reassuring that we found similar long-term outcomes in the bedaquilinedelamanid combination group despite this group having a higher frequency of poor prognostic features; at least half of the group had experienced MDR treatment failure compared to $\sim 10 \%$ in the bedaquiline group, and almost a quarter of isolates in the combination group were resistant to five or more drugs compared to $\sim 5 \%$ in the bedaquiline-only group. Furthermore, usage of WHO group A and group B drugs (i.e. linezolid, any fluoroquinolone, clofazimine and terizidone) were similar in both groups. Despite recent findings suggesting limited efficacy of this drug (and when using 6-month culture conversion as an outcome) [16], our data suggest that delamanid may be a useful addition in patients for whom an appropriate regimen of four to five likely effective drugs cannot be constituted because of toxicity or high-level resistance. Indeed, despite the combination group having an $\sim 50 \%$ prior treatment failure rate, the 6-month culture conversion rate in this group was $>80 \%$ and the overall long-term favourable outcome rate was almost $70 \%$. This far exceeds the dismal outcomes seen with XDR-TB prior to the advent of newer drugs [11, 25]. Nevertheless, $\sim 10 \%$ of patients in each group failed treatment. Thus, programmatically incurable TB is an emerging problem in TB-endemic countries and public health efforts are needed to manage such patients on a long-term basis. Besides establishing palliative care and long-term community-based residential facilities [26], preventative measures such as optimal antibiotic stewardship, active case finding, and wider roll-out of new diagnostics and drugs are urgently required [2].

In HIV-infected patients the adverse event profile and treatment outcomes showed similar patterns compared to HIV-uninfected patients. These findings are highly relevant to high TB- and HIV-endemic settings. Besides QT prolongation, other important adverse events, such as elevation of liver enzymes, were found to be similar in the HIV-infected patients in both groups, further confirming safety and compatibility with antiretrovirals in this group.

There are several limitations of our findings. Our study was of limited sample size and a larger study may have shown different results given that cardiovascular events related to QT prolongation are rare. A larger sample size may have also allowed some clear-cut outcome effect to be discerned. However, this study has reported the largest number of patients on bedaquiline-delamanid combination therapy $(n=40)$ and the largest number of patients with XDR-TB $(n=86)$, and the multivariable analysis, even when taking into account the poorer prognostic features in the combination group, failed to identify the combination as an independent predictor of outcome. Rather, bacterial load remained the only significant and independent predictor of outcome. This highlights another limitation, which is the failure to evaluate radiographic disease extent at the time of diagnosis, which would better enable us to account for initial disease severity. However, logistical and technical issues prevented us from accessing the pre-treatment chest radiographs. Nevertheless, we were able to get a fairly good comparative estimation of disease severity in both groups through evaluation of other prognostic features such as admission weight, HIV status, CD4 count, previous treatment history, resistance to five or more drugs and mycobacterial load, which are all proxies of disease severity. Selection bias could have also impacted our findings, including the cases and controls study design. However, all the patients were prospectively recruited in the same region and over a similar timeframe. It is possible that some patients with events could have been missed as the programme in the Western Cape became more decentralised. However, our recruitment network spanned the entire region and also utilised a region-wide electronic capture and surveillance system.

In summary, our findings suggest that a bedaquiline-delamanid combination has a comparable long-term safety profile to a bedaquiline-based regimen in patients with DR-TB, irrespective of HIV status. Delamanid appeared to be a useful adjunct in the treatment of patients with poor prognostic features or 
high-level resistance where constituting an appropriate regimen would otherwise be challenging. These data inform regimen selection in patients with DR-TB from TB-endemic settings.

Conflict of interest: None declared.

Support statement: This work was funded by South African Medical Research Council (grant: RFA-EMU-02-2017) and European and Developing Countries Clinical Trials Partnership (grant: TMA-2015SF-1043 and TMA-1051-TESAII).

Funding information for this article has been deposited with the Crossref Funder Registry.

\section{References}

1 Global Tuberculosis Report 2018. Geneva, World Health Organization, 2018

2 Dheda K, Gumbo T, Maartens G, et al. The epidemiology, pathogenesis, transmission, diagnosis, and management of multidrug-resistant, extensively drug-resistant, and incurable tuberculosis. Lancet Respir Med 2017; 5: 291-360.

3 Dheda K, Barry CE, Maartens G. Tuberculosis. Lancet 2016; 387: 1211-1226.

4 WHO Position Statement on the Use of Delamanid for Multidrug-Resistant Tuberculosis. Geneva, World Health Organization, 2018.

5 WHO Consolidated Guidelines on Drug-Resistant Tuberculosis Treatment. Geneva, World Health Organization, 2019.

6 Collaborative Group for the Meta-Analysis of Individual Patient Data in MDR-TB treatment-2017, Ahmad N, Ahuja SD, et al. Treatment correlates of successful outcomes in pulmonary multidrug-resistant tuberculosis: an individual patient data meta-analysis. Lancet 2018; 392: 821-834.

7 Olayanju O, Limberis J, Esmail A, et al. Long-term bedaquiline-related treatment outcomes in patients with extensively drug-resistant tuberculosis from South Africa. Eur Respir J 2018; 51: 1800544.

8 Schnippel K, Ndjeka N, Maartens G, et al. Effect of bedaquiline on mortality in South African patients with drug-resistant tuberculosis: a retrospective cohort study. Lancet Respir Med 2018; 6: 699-706.

9 Diacon AH, Pym A, Grobusch MP, et al. Multidrug-resistant tuberculosis and culture conversion with bedaquiline. N Engl J Med 2014; 371: 723-732.

10 Lee M, Lee J, Carroll MW, et al. Linezolid for treatment of chronic extensively drug-resistant tuberculosis. $N$ Engl J Med 2012; 367: 1508-1518.

11 Dheda K, Limberis JD, Pietersen E, et al. Outcomes, infectiousness, and transmission dynamics of patients with extensively drug-resistant tuberculosis and home-discharged patients with programmatically incurable tuberculosis: a prospective cohort study. Lancet Respir Med 2017; 5: 269-281.

12 Casali N, Nikolayevskyy V, Balabanova Y, et al. Evolution and transmission of drug-resistant tuberculosis in a Russian population. Nat Genet 2014; 46: 279-286.

13 Shah NS, Auld SC, Brust JC, et al. Transmission of extensively drug-resistant tuberculosis in South Africa. $N$ Engl J Med 2017; 376: 243-253.

14 Dheda K, Gumbo T, Gandhi NR, et al. Global control of tuberculosis: from extensively drug-resistant to untreatable tuberculosis. Lancet Respir Med 2014; 2: 321-338.

15 Olayanju O, Esmail A, Limberis J, et al. Linezolid interruption in patients with fluoroquinolone-resistant tuberculosis receiving a bedaquiline-based treatment regimen. Int J Infect Dis 2019; 85: 74-79.

16 von Groote-Bidlingmaier F, Patientia R, Sanchez E, et al. Efficacy and safety of delamanid in combination with an optimised background regimen for treatment of multidrug-resistant tuberculosis: a multicentre, randomised, double-blind, placebo-controlled, parallel group phase 3 trial. Lancet Respir Med 2019; 7: 249-259.

17 Mohr E, Hughes J, Reuter A, et al. Delamanid for rifampicin-resistant tuberculosis: a retrospective study from South Africa. Eur Respir J 2018; 51: 1800017.

18 Skripconoka V, Danilovits $\mathrm{M}$, Pehme L, et al. Delamanid improves outcomes and reduces mortality in multidrug-resistant tuberculosis. Eur Respir J 2013; 41: 1393-1400.

19 Lewis JM, Sloan DJ. The role of delamanid in the treatment of drug-resistant tuberculosis. Ther Clin Risk Manag 2015; 11: 779-791.

20 Pontali E, Sotgiu G, Tiberi S, et al. Cardiac safety of bedaquiline: a systematic and critical analysis of the evidence. Eur Respiratory Soc 2017; 50: 1701462.

21 The Use of Delamanid in the Treatment of Multidrug-Resistant Tuberculosis: Interim Policy Guidance. Geneva, World Health Organisation, 2014.

22 Ferlazzo G, Mohr E, Laxmeshwar C, et al. Early safety and efficacy of the combination of bedaquiline and delamanid for the treatment of patients with drug-resistant tuberculosis in Armenia, India, and South Africa: a retrospective cohort study. Lancet Infect Dis 2018; 18: 536-544.

23 Kim CT, Kim TO, Shin HJ, et al. Bedaquiline and delamanid for the treatment of multidrug-resistant tuberculosis: a multicentre cohort study in Korea. Eur Respir J 2018; 51: 1702467.

24 Pontali E, Sotgiu G, Tiberi S, et al. Combined treatment of drug-resistant tuberculosis with bedaquiline and delamanid: a systematic review. Eur Respir J 2018; 52: 1800934.

25 Pietersen E, Ignatius E, Streicher EM, et al. Long-term outcomes of patients with extensively drug-resistant tuberculosis in South Africa: a cohort study. Lancet 2014; 383: 1230-1239.

26 Dheda K, Migliori GB. The global rise of extensively drug-resistant tuberculosis: is the time to bring back sanatoria now overdue? Lancet 2012; 379: 773-775. 